\title{
The Prophylaxis Potential of Carnosine in the Management of COVID-19
}

\author{
Charulata Jindal and Jimmy Efird *
}

\begin{abstract}
After Severe Acute Respiratory Syndrome Coronavirus (SARS-CoV) and Middle East Respiratory Syndrome Coronavirus (MERS-CoV), Severe Acute Respiratory Syndrome Coronavirus-2 (SARS-CoV-2) - the causative agent of the disease known as COVID-19-is the third coronavirus to pose serious threats to human society in this century. With $67,367,046$ confirmed cases and 1,545,331 deaths worldwide as of 10 December 2020, this disease has become a global emergency. Mortality rates are higher among the elderly population and those with comorbidities such as cancer, diabetes, cardiovascular problems, and hypertension obesity. Increased oxidative stress/cytokine storm is also one of the main causes of COVID-19-associated complications. Carnosine is a well-established anti-inflammatory, anti-aging, antihypertensive, antineoplastic, antichelating, and neuroprotective agent that is helpful in maintaining the body's pH balance. It is a naturally occurring dipeptide and is present in human skeletal muscles, cardiac muscles, and brain. The compound reduces the concentration of oxygen free radicals; chelates heavy metals such as iron, zinc, and copper; and inhibits protein glycation and the generation of protein carbonyls. The carnosine/non-hydrolyzed isopeptide of carnosine/N-acetylcarnosine, which is available without prescription, merits further consideration as a low-cost prophylactic approach to COVID-19 and the associated complications of this disease. This is especially relevant to low-resource regions of the world, which may be the last to achieve population-based herd immunity through vaccination.
\end{abstract}

Keywords: carnosine; COVID-19; anti-inflammatory; anti-aging; antihypertensive; antineoplastic; antichelating; neuroprotective

Publisher's Note: MDPI stays neu-

tral with regard to jurisdictional

claims in published maps and insti-

tutional affiliations.

\section{(c) (i)}

Copyright: (c) 2020 by the authors. Submitted for possible open access publication under the terms and conditions of the Creative Commons Attribution (CC BY) license (http://creativecommons.org/licenses /by/4.0/). 